\title{
Leiomyoma with degeneration mimicking an ovarian neoplasm
}

\begin{abstract}
Leiomyoma of the uterus is the most common tumor of the female pelvis and is seen in nearly half of the women above age 35 . Large myomas with degenerations located towards the annexed may mimic an ovarian neoplasm and can impose a challenge in its diagnosis. We report an unusual case of a large, cystic, pedunculated uterine leiomyoma mimicking an ovarian tumor which misled the radiologist and the surgeon pre-operatively. As leiomyomas enlarge, they can outgrow their blood supply, resulting in various types of degeneration, such as hyaline, cystic, myxoid or red degeneration and dystrophic calcification. 3 Hyalinization is the most common type of degeneration, occurring in up to $60 \%$ of cases. Cystic degeneration is observed in about $4 \%$ of leiomyomas. Our case showed leiomyoma with both the secondary changes being reported on histopathology. The large size of the myoma, associated degeneration and its growth from the uterus towards the broad ligament laterally mimicked it to be an ovarian neoplasm. Fibroids masquerading as ovarian tumors impose a challenge to both the radiologist and the surgeon and should always be in the list of differentials. Magnetic resonance imaging although not the first choice, may be used if ultrasonography is inconclusive. However a stepwise and a multidisciplinary approach in management of these cases is preferred to achieve optimal results.
\end{abstract}

Volume 2 Issue 3 - 2017

\author{
Esha Khanuja, Mamta Tyagi, Smriti Gupta, \\ Ruby Yadav \\ Department of Gynecologist \& Obstetrician Doctors, Subharti \\ medical college, India
}

\begin{abstract}
Correspondence: Esha Khanuja, Deportment of Gynecologist \& Obstetrician Doctors, Subharti medical college, delhi haridwar bypass road, Subhartipuram, meerut-250005, India, Tel
\end{abstract} 9675660336,Email dreshakhanuja@gmail.com

Received: December 14, 2016 | Published: May 04, 2017

Keywords: leiomyoma, neoplasm, degeneration, ultrasonography, mri

\section{Introduction}

Leiomyoma of the uterus is the most common tumor of the female pelvis, arising from the uterine smooth muscle. Its prevalence increases during the reproductive age and is seen in nearly half of the women over the age of $35 .{ }^{1}$ Large myomas with degenerations located towards the adnexa may mimic an ovarian neoplasm and can impose a challenge in its diagnosis. Radiological modalities like ultrasonography and MRI may aid in differentiating the two. We report an unusual case of a large, cystic, pedunculated uterine leiomyoma mimicking an ovarian tumor which misled the radiologist and the surgeon pre-operatively.

\section{Case report}

A 30year multiparous female came to gynecology out-patient department with complaint of pain in left flank, heaviness and progressively increasing lump abdomen since one and half years which was initially felt below the umbilicus and had increased to the present size in last 6months. There was no family history of ovarian, breast or colorectal cancer. She was a known hypertensive on medication and had past renal disease of which documents were unavailable. No other significant past history was present. There was no abnormality in menstrual cycles. On general examination vitals were stable, mild pallor was present with no lymphadenopathy. Systemic examination was normal. On abdominal examination, a lump of variable consistency corresponding to 34weeks of gravid uterus was felt which occupied hypogastria, umbilical, right and left lumbar and epigastria area. The lower limit of the lump could not be reached and had restricted mobility. On internal examination, same lump of variable consistency was felt through all the fornices. Blood and urine investigations were sent of which $\mathrm{Ca} 125$ was 5.87, blood urea and serum creatinine were 48.10 and 2.1 respectively. Urinary albumin was $2+$. Ultrasound revealed that uterus was displaced interiorly and compressed by the mass with non-visualization of both ovaries. A large mass lesion extending from pelvis to epigastria area with heteroechoeic echo texture, cystic and solid areas and mild internal vascularity was seen. MRI report revealed multilobulated pelvic abdominal solid cystic lesion likely to be ovarian neoplasm (Figure 1).

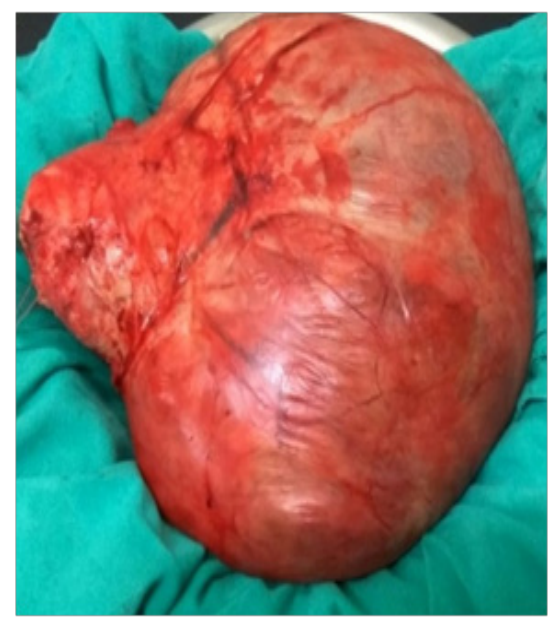

Figure I Image showing leiomyoma with cystic degeneration.

After anesthesia clearance patient was taken for laparotomy. Per-operatively minimal free fluid present was sent for cytological examination. A huge solid mass of around $30 \times 28 \mathrm{~cm}$ with cystic degeneration was seen. Right ovary visualized was grossly normal. The funds of the uterus was visible and the left ovary could not be visualized due to the large mass. The lump was exteriorized and was 
then seen arising from left lateral surface of body of uterus. Left ovary which was masked by the lump was now visible and appeared normal. Considering the age and fertility of the patient decision of myomectomy was taken. The mass was approached by opening the leaves of broad ligament. The lump grossly appeared benign due to its variegated consistency, cystic and solid areas with mild vascularity. The blood vessels on the surface were congested. The lesion was delivered after clamping its stalk. The large defect in leaves of broad ligament was sutured. Ureter was traced and peristalsis observed before closure. Cytology of intraperitoneal free fluid revealed no malignant cells. Histopathology of the excised tumor showed features of leiomyoma with extensive hydropic degeneration. Patient was kept on regular follow up till date.

\section{Discussion}

Leiomyomas are classified as sub mucosal, intramural or subserosal. ${ }^{2}$ The latter may be pedunculated and then simulate ovarian neoplasm as was seen in our case. As leiomyomas enlarge, they can outgrow their blood supply, resulting in various types of degeneration, such as hyaline, cystic, myxoid or red degeneration and dystrophic calcification. ${ }^{3}$ Hyalinization is the most common type of degeneration, occurring in up to $60 \%$ of cases. Cystic degeneration, observed in about $4 \%$ of leiomyomas, may be considered extreme squeal of edema. ${ }^{4}$ Our case showed leiomyoma with both the secondary changes being reported on histopathology. The large size of the myomas, associated degeneration and its growth from the uterus towards the broad ligament laterally mimicked it to be an ovarian neoplasm. Additionally on radiological examination ultrasound revealed a large lesion displacing the uterus along with non-visualization of both ovaries and MRI too claimed it to be likely of ovarian origin. This potential of uterine leiomyomas to grow to an extreme size before causing symptoms is quite remarkable. It is likely due to the relatively large volume of the abdominal cavity, the distensibility of the abdominal wall and the slow growth rate of these tumors. ${ }^{5}$ Typical appearances of leiomyomas are easily recognized on imaging. However, the atypical appearances that follow degenerative changes can cause confusion in diagnosis as was in our case. Although Magnetic resonance imaging is more accurate in differentiating leiomyomas from ovarian masses and maybe used when ultrasonography is inconclusive ${ }^{1}$, but it was not beneficiary in this case mostly due its large size and its growth laterally towards the broad ligament which masked the ovaries behind and led to the confusion.

\section{Conclusion}

Fibroids masquerading as ovarian tumors impose a challenge to both the radiologist and the surgeon. A large myomas with secondary changes may mimic an ovarian neoplasm and thus should always be in the list of differentials. Magnetic resonance imaging although not the first choice, may be used if ultrasonography is inconclusive. However a stepwise and a multidisciplinary approach in management of these cases are preferred to achieve optimal results.

\section{Acknowledgements}

None.

\section{Conflict of interest}

Author declares that there is no conflict of interest.

\section{References}

1. Monappa Geeth. Leiomyomas Masquerading As Ovarian Neoplasms. IOSR Journal of Dental and Medical Sciences. 2015;14(7):5-9.

2. Novak ER, Woodruff JD. In: Novak ER, Woodruf JD, editors. Novak's gynecologic and obstetric pathology. WB Saunders, Philadelphia; 1979. p. 260-279.

3. Preayson RA, Hart WR. Pathologic considerations of uterine smooth muscle tumors. Obstet Gynecol Clin North Am. 1995;22(4):637-657.

4. Mayer DP, Shipilov V. Ultrasonography and magnetic resonance imaging of uterine fibroids. Obstet Gynecol Clin North Am. 1995;22(4):667-725.

5. Aydin C, Eriş S, Yalçin Y, et al. A giant cystic leiomyoma mimicking an ovarian malignancy. Int J surg case rep. 2013;4(11):1010-1012. 